\title{
A self-report questionnaire to detect hand dermatitis in nurses
}

Author: V Parsons ${ }^{1}, \mathrm{H}$ C Williams², J English ${ }^{3}$, J Llewellyn², G Ntani ${ }^{4}$, I Madan $^{1}$

1. Occupational Health Service, Guy's and St Thomas NHS Foundation Trust / Faculty of Life Sciences and Medicine, King's College London, London, UK

2. Centre of Evidence Based Dermatology, University of Nottingham, Nottingham, UK

3. Dermatology, Circle Nottingham NHS Treatment Centre, Nottingham, UK

4. Medical Research Council Lifecourse Epidemiology Unit, University of Southampton, Southampton, UK

Correspondence to:

V. Parsons, Occupational Health Service, Guy's and St Thomas NHS Foundation Trust, St Thomas Hospital, Westminster Bridge Road, London UK SE1 7NJ email: Vaughan.parsons@gstt.nhs.uk 


\section{A self-report questionnaire to detect hand dermatitis in nurses}

\section{Abstract}

Background Hand dermatitis is highly prevalent among nurses due to their frequent exposure to wet work. Providing cost-effective dermatological health surveillance for this occupational group presents a challenge to health service providers.

Aim To ascertain the predictive value of nurses' self-assessment of whether they had current hand dermatitis using a screening questionnaire when compared with the assessment made by a dermatologist of the nurses' hand photographs.

Methods We conducted a cross-sectional study comparing the self-report decision made by student and intensive care nurses using a single hand dermatitis screening question with the clinical assessment of their hand photographs made by dermatologists using a standardised photographic guide.

Results We analysed data collected at study baseline $(n=1599)$. The results showed that the screening question had a high negative predictive value (91\%; $95 \% \mathrm{Cl} 89-93))$, but a low positive predictive value $(39 \% ; 95 \% \mathrm{Cl} 34-45)$. It demonstrated acceptable accuracy in distinguishing those with and without the disease (area under the receiver operator curve $=0.7)$ and had a high specificity $(86 \%$; $\mathrm{Cl} 84-88))$ but a sensitivity of only $52 \%(\mathrm{Cl} 46-$ 59)in identifying hand dermatitis.

Conclusion We found that nurses were able to accurately self-assess themselves as not having any signs of hand dermatitis. By contrast, they were less able to accurately selfassess positive cases suggesting under-recognition of early disease. We propose that a questionnaire containing a single hand dermatitis screening question should be considered 
as a tool for screening out clear cases as part of a workplace health surveillance programme for detecting hand dermatitis.

Key words: Hand dermatitis, hand photographs, health surveillance, occupational health, questionnaire, self-report, - 


\section{Introduction}

Hand dermatitis is more prevalent in healthcare workers than the general population $(21 \%$ versus $10 \%$ respectively) [1]. Healthcare workers who work in clinical roles which involve frequent hand-washing and prolonged glove use are at increased risk of developing irritant contact dermatitis [2]. In the United Kingdom, the Health and Safety Executive (HSE) recommends that employers ensure that clinical health care workers are provided with regular health surveillance for hand dermatitis [3]. The HSE suggests that the surveillance might include skin inspections by a 'competent person' or annual self-reported employee questionnaires. The introduction of health surveillance programmes is expensive and little is known about how effective they are at detecting hand dermatitis. We do not know the diagnostic accuracy of dermatological assessments made by non-dermatology trained healthcare workers [4]. This cross-sectional study assessed the diagnostic accuracy of student and intensive care nurses' self-assessment of hand dermatitis with diagnosis by dermatologists using hand photographs, using data obtained from the skin care intervention in nurses (SCIN) trial [5]. In this multi-centre randomised controlled trial, we tested the clinical- and cost-effectiveness of a behaviour change intervention to improve hand care and coupled with provision of hand moisturising cream to reduce the prevalence of hand dermatitis in at-risk nurse.

\section{Methods}

There were two groups of participants in the SCIN trial i) student nurses about to start their first clinical placement and who were at increased risk of hand dermatitis because of a history of atopic disease; and ii) full-time intensive care unit (ICU) nurses who were at increased risk of hand dermatitis through workplace exposure. All participants who took part in the SCIN trial $(n=2040)$ were invited to complete a short questionnaire at baseline and at 12 months, based on the Nordic Occupational Short Skin Questionnaire (NOSQ). Specifically, the HOSQ is a questionnaire for screening and monitoring occupational skin diseases and includes questions on demographics and occupation (job role and hours 
workers), self-report history and duration of hand eczema and exposures. In the HOSQ tool, questions relating to the self-assessment of dermatitis are expressed as 'Have you ever had hand eczema? When did you last have hand eczema?' Whereas in our study specific questionnaire, we asked participants a series of slightly modified questions which included 'In your opinion, do you currently have hand / wrist dermatitis?', 'Where is your hand/wrist dermatitis or skin condition located?' and 'How long have you had the current skin condition?' [6].

The self-assessment questionnaire included a two-part question: a) 'In your opinion, do you currently have hand/wrist dermatitis'? The response options were yes/no/unsure. This was followed by the second part of the question b) 'where is your hand/wrist dermatitis or skin condition located? The participants were given an option of eight boxes and where asked to tick all those that applied. The options were: left hand palm; left hand back; left wrist (front or back); the same options of these three locations for the right hand; between the fingers, and other with a request to specify site if this category was selected. We categorised unsure as "yes" if they reported having dermatitis in any of left/right palm, right/left back of the hand, right/left wrist, between fingers, and it was recoded to "no" if they said "no" to all left/right palm, right/left back of the hand, right/left wrist, between fingers. On the same day as participants completed the questionnaire, photographs of the palm and dorsum aspects of both hands and wrists were taken using a standardised method [2]. The hand photographs were assessed by the trial dermatologists using a validated method described elsewhere [7]. For instance, each of the two dermatologists assessed the hand photographs independently, and they reported for each of four parts of the hands (right hand at the dorsum, right hand in the palm, left hand at the dorsum and left hand in the palm) whether there was clear, almost clear, moderate, severe, or very severe sings of dermatitis. Then the maximum score (clear, almost clear, moderate, severe, very severe) was taken across those four hands' parts. If both dermatologists agreed that all four parts of the hands were clear (i.e. no sign of dermatitis), then the participant was categorised as negative for dermatitis. If both 
dermatologists agreed that any part of the hand had signs of dermatitis (either almost clear, moderate, severe, or very severe), then the participant was categorised as positive for dermatitis. The photographs for which assessment did not agree between the two dermatologists (i.e. one considered that the hand was "clear" and the other that the hand was at least "almost clear"), were sent back to the dermatologists for a joint assessment. In case of disagreement between the two dermatologists' assessment on the final dichotomous outcome, the corresponding photographs were sent to them again for joint assessment.[5]. Agreement between self-assessed presence of dermatitis compared with dermatologistassessed presence of dermatitis was described using sensitivity, specificity, and receiver operator curve (ROC) analyses. Analyses were carried out using Stata version 12 [8]. Ethics approval was granted by the Health Research Authority (reference 13/LO/0981).

\section{Results}

Dermatologists conducted dermatitis assessments on 1,966 nurses using hand photographs of participants. In addition, 1,674 nurses returned questionnaires in which they self-assessed and self-reported the presence or absence of dermatitis. Rates of baseline and follow-up dermatitis were similar between those included and those excluded from the study (data not shown). A total of 1,599 nurses had usable baseline dermatologist dermatitis assessments with no missing data on the relevant question in the baseline questionnaire. In this sample, the median age of nurses was 28 years (IQR: 23-39) with the majority being females (89\%). Table 1 presents the agreement of the presence of hand/wrist dermatitis as assessed by the study dermatologists compared with self-reported hand/ wrist dermatitis at baseline. The findings were similar in student and ICU nurses (table 2), showing an overall high negative predictive value of $91 \%(95 \% \mathrm{Cl} 89-93 \%)$ and a low positive predictive value of $39 \%(95 \% \mathrm{Cl}$ $34-45 \%)$ with a corresponding high degree of specificity, $86 \%$ ( $95 \% 84-88 \%$. The point prevalence of hand dermatitis in all our participants was $15 \%(95 \% \mathrm{Cl}: 13 \%$ to $17 \%)$, in student nurses $13 \%$ (95\% Cl: $10 \%$ to $16 \%)$, and in ICU nurses $16 \%(95 \% \mathrm{Cl}$ : $14 \%$ to $19 \%)$. The ROC area is used to illustrate the trade-off between the sensitivity and specificity of our 
screening question in detecting hand dermatitis. The area under the ROC curve is a measure of the performance of a test, where a greater area indicates a more useful test. In our study, the ROC area was 0.7 , which means that our screening question has a $70 \%$ chance of distinguishing between positive and negative cases of hand dermatitis.

\section{Discussion}

Results showed the negative predictive value of our single question was high, that is participants were able to accurately assess themselves as not having hand dermatitis The specificity of the question was higher than the sensitivity, suggesting that our question underestimates rather than overestimates the point prevalence of hand dermatitis. The results were similar for student nurses before they started their clinical work as for experienced ICU nurses.

A particular strength of this study is that it benefited from having many participants with dermatologist assessed hand photographs and self-assessed questionnaire. Our reference standard of two consultant dermatologists with a special interest in eczema was strong, and although evaluating images is not the same as examining participants in person, the quality of the images were good. Possible reasons for discordant results especially in terms of lower sensitivity include under-recognition of early signs of dermatitis by nurses, denial of hand dermatitis by nurses and increased recognition of mild dermatitis by dermatologists who could magnify the images. The majority of our participants were women and this reflects the composition of the nursing workforce in terms of the female to male ratio. An important limitation with this study is that we cannot confidently extrapolate our results beyond nurses with a similar point prevalence of dermatitis. Furthermore, the results are not generalisable to healthcare workers outside the nursing profession or to other workers groups (e.g. construction workers or hairdressers). 
The findings are supported by those of Bregnhøj et al's [9] study of hairdresser apprentices, which also showed that self-assessment of presence or absence of hand dermatitis (with skin examination by dermatologists) had a high negative predictive value of $98.8 \%$. Participants in the Bregnhøj study were younger (mean age 17.5 years) compared with participants in our study, with lower point prevalence of hand dermatitis (4.8\%), but like in our study, most participants were females (95\%). A Swedish study of dentists and office workers attending an eczema clinic and participants of an epidemiological study (whose inclusion criteria was to have hand dermatitis in the last 12 months) found that the negative predictive value of asking ' do you currently have hand eczema/dermatitis' was high, $87.2 \%$ [10]. The gold standard was a hand examination by a dermatologist and the single question had a higher negative predictive value than a symptom-based questionnaire [10]. Our findings that the specificity of a self-report question was higher than the sensitivity in detecting hand dermatitis has been found in other studies involving healthcare workers [6, 11]. The results provide a compelling case for undertaking a study evaluating the use of a self-report screening questionnaire containing our single self-assessment dermatitis question in workplace hand dermatitis health surveillance programmes targeting nurses and other healthcare workers If such a tool were practicable to deliver and proves to be a clinically reliable and cost-effective method of screening out 'clear' (no hand dermatitis) cases, further action would only be necessary if positive cases are reported.

\section{Key learning points:}

\section{What is already known about this subject:}

- The prevalence of irritant contact hand dermatitis is higher among healthcare workers than the general population

- A range of methods have been developed to screen and diagnose hand dermatitis ranging from self-assessment questionnaires through to clinical examination. 


\section{What this study adds:}

- This is the first study to compare the diagnostic accuracy of self-report measures of hand dermatitis by nurse participants with diagnosis from clinical examinations of hand photographs by dermatologists.

- The results show that nurses can competently use a single item question to selfassess the absence of hand dermatitis in themselves.

\section{What impact this may have on practice or policy:}

- The results from this study are likely to have important implications for clinical practice notably strengthening the case for workplace health surveillance and for future epidemiological studies.

- Incorporating a single-item, self-assessed question as part of health surveillance should be formally evaluated in a range of different healthcare workers, and if found to be effective at screening out 'clear' cases, could be an important component of dermatitis health surveillance programmes in hospitals.

Acknowledgement: The authors acknowledge the contribution made by the SCIN trial coinvestigators: David Coggon, Barry Cookson, Tina Lavender, Paul McCrone, Caroline Murphy, Lesley Rushton, Julia Smedley and Alison Wright.

Funding: This study is funded by the National Institute for Health Research (NIHR) Health Technology Assessment Programme (project reference 13/LO/0981). The views expressed are those of the author(s) and not necessarily those of the NIHR or the Department of Health and Social Care. 


\section{References}

1. Thyssen JP, Johansen JD, Linneberg A, Menné T. The epidemiology of hand eczema in the general population - Prevalence and main findings. Contact Dermatitis. 2010;62(2):75-87.

2. Madan I, Parsons V, Ntani G, Coggon D, Wright A, English J, et al. A behaviour change package to prevent hand dermatitis in nurse working in the National Health Service: results of a cluster randomised controlled trial. The British journal of dermatology. 2020.

3. Health \& Safety Executive. Health surveillance.: Health and Safety Executive; 202003 February 2020 [Available from: https://www.hse.gov.uk/skin/professional/healthsurveillance.htm.

4. Tran H, Chen K, Lim A, Jabbour J, Shumack S. Assessing diagnostic skill in dermatology: A comparion between general practitioners and dermatologists. Australian Journal of Dermatology. 2005;46:230-4.

5. Madan I, Parsons V, Ntani G, Wright A, English J, Coggon D, et al. A behaviour change package to prevent hand dermatitis in nurses working in health care: the SCIN cluster RCT. Health Technol Assess. 2019;23(58):1-92.

6. Susitaival P, Flyvholm M, Meding B, Kanerva L, Lindberg M, Svensson Å, et al. Nordic occupational skin questionnaire (NOSQ-2002): a new tool for surveying occupational skin diseases and exposure. Contact Dermatitis. 2003;49(2):70-6.

7. Parsons V, Williams C, English J, Llewellyn J, Ntani G, Madan I. The development of a protocol for diagnosing hand dermatitis from photographic images. Contact Dermatitis. 2018:1-6.

8. StataCorp. Stata Statistical Software: Release 12. College Station, TX: StataCorp LP.2011.

9. Bregnhøj A, Søsted H, Menné T, Johansen JD. Validation of self-reporting of hand eczema among Danish hairdressing apprentices. Contact Dermatitis. 2011;65(3):14650.

10. Svensson A, Lindberg M, Meding B, Sundberg K, Stenberg B. Self-reported hand eczema: symptom-based reports do not increase the validity of diagnosis. The British journal of dermatology. 2002;147(2):281-4.

11. Nichol K, Copes R, Kersey K, Eriksson J, Holness DL. Screening for hand dermatitis in healthcare workers: Comparing workplace screening with dermatologist photo screening. Contact Dermatitis. 2019;80(6):374-81. 


\begin{tabular}{|c|c|c|c|c|}
\hline & & \multicolumn{2}{|c|}{$\begin{array}{c}\text { Hand dermatitis assessed by } \\
\text { dermatologists from hand } \\
\text { photographs (baseline) }\end{array}$} & \multirow[b]{2}{*}{ Total } \\
\hline & & No & Yes & \\
\hline \multirow[t]{3}{*}{$\begin{array}{l}\text { Self-reported hand } \\
\text { dermatitis } \\
\text { (baseline) }\end{array}$} & No & $\begin{array}{c}\mathrm{n}=1,166(73 \%) \\
\text { (True negative } \\
(T N))\end{array}$ & $\begin{array}{c}\mathrm{n}=115(7 \%) \\
(\text { False negative } \\
(F N))\end{array}$ & $\mathrm{n}=1,281(80 \%)$ \\
\hline & Yes & $\begin{array}{l}\mathrm{n}=193(12 \%) \\
\text { (False positive } \\
(F P))\end{array}$ & $\begin{array}{c}\mathrm{n}=125(8 \%) \\
\text { (True positive } \\
(T P))\end{array}$ & $\mathrm{n}=318(20 \%)$ \\
\hline & Total & $\mathrm{n}=1,359(85 \%)$ & $\mathrm{n}=240(15 \%)$ & $\mathrm{n}=1,599(100 \%)$ \\
\hline
\end{tabular}


Table 2: The specificity, sensitivity, and predictive value of self-reported dermatitis by participant group.

\begin{tabular}{|l|c|c|c|}
\hline & $\begin{array}{c}\text { All } \\
(\mathbf{9 5 \%} \mathbf{~ C l})\end{array}$ & $\begin{array}{c}\text { Student Nurses } \\
(\mathbf{9 5 \%} \mathbf{~ C l})\end{array}$ & $\begin{array}{c}\text { ICU Nurses } \\
(\mathbf{9 5 \%} \text { CI) }\end{array}$ \\
\hline Sensitivity & $52 \%(46-59)$ & $52 \%(41-62)$ & $52 \%(44-61)$ \\
\hline Specificity & $86 \%(84-88)$ & $87 \%(84-90)$ & $85 \%(82-87)$ \\
\hline ROC area & $0.7(0.7-0.7)$ & $0.7(0.6-0.7)$ & $0.7(0.6-0.7)$ \\
\hline Positive predictive value & $39 \%(34-45)$ & $37 \%(29-47)$ & $41 \%(34-48)$ \\
\hline $\begin{array}{l}\text { Negative predictive } \\
\text { value }\end{array}$ & $91 \%(89-93)$ & $92 \%(90-94)$ & $90 \%(88-92)$ \\
\hline
\end{tabular}

